\title{
Study of electrical properties of piezoresistive pastes and determination of the electrical transport
}

\author{
S. Vionnet ${ }^{1}$, C. Grimaldi ${ }^{1}$, T. Maeder ${ }^{1,2}$, P. Ryser ${ }^{1}$, and S. Strässler ${ }^{1,2}$ \\ ${ }^{1}$ Laboratoire de Production Microtechnique, IPR, Ecole Polytechnique Fédérale Lausanne, Lausanne, \\ Switzerland \\ ${ }^{2}$ Sensile Technologies SA, PSE, CH-1015 Lausanne, Switzerland
}

Version of record: Journal of the European Ceramic Society 25 (12), 2129-2132, 2005. http://hdl.handle.net/10.1016/j.jeurceramsoc.2005.03.018

\begin{abstract}
Thick-film resistors (TFRs) consist of a percolating network of conducting oxide nanoparticles dispersed in an insulating glassy matrix, whose resistive properties are dominated by quantum tunneling across insulating layers separating adjacent conducting grains. Tunneling processes are at the origin of the high sensitivity of the TFRs resistances to applied strains. In this work, we aim to define the electrical transport law between metallic nanoparticles in piezoresistive pastes. We have measured transport and piezoresistive response for different $\mathrm{RuO}_{2}$-based TFRs as a function of metallic concentration $\mathrm{x}$ and $\mathrm{RuO}_{2}$ grain sizes.

The study reveals that the conductivity is shown to vanish as $\mathrm{x}$ approaches a critical concentration $\mathrm{x}_{\mathrm{c}}$ by following a power law with nonuniversal critical exponents, while the piezoresistivity diverges at the same critical concentration. We argue that nonuniversality and diverging piezoresistivity have the same origin and arise from the highly fluctuation inter-grain tunneling distances determined by the segregated microstructure of TFRs.
\end{abstract}

Keywords: B: Nanocomposites, C: Electrical conductivity. E: Sensors.

\section{INTRODUCTION}

Thick-film resistors (TFRs) have found a widespread use in the field of pressure and force sensors due to their low cost, good stability, and high piezoresistive response. ${ }^{1,2}$ The typical microstructure of TFRs is characterised by an insulating glassy phase embedding randomly dispersed submicron conducting grains (usually $\mathrm{RuO}_{2}$ or ruthenates such as $\mathrm{Pb}_{2} \mathrm{Ru}_{2} \mathrm{O}_{7}$ ). The dominant mechanism of transport is via quantum tunnelling through the nanometer-thick film of glass separating two neighbouring conducting particles. ${ }^{3,4,5}$ The macroscopic transport properties are then governed by tunnelling paths percolating the entire sample, giving rise to a strong dependence upon the metallic volume fraction. ${ }^{6}$ Here we show that the percolative properties strongly depend upon the tunnelling parameters, which can be modified in a controlled way by applying an external strain $\varepsilon$ in piezoresistive measurements. In particular, we have 
found that the critical exponent $t$ characterising the metal-insulator transition is affected by $\varepsilon$, giving evidence of a tunnelling-distance dependence of $t$.

\section{EXPERIMENTAL}

We have fabricated several series of TFRs by dispersing in an organic vehicle (terpineol and ethyl-cellulose) $\mathrm{RuO}_{2}$ powders and a lead-borosilicate glass frit $\left[\mathrm{PbO}(75 \% \mathrm{wt})-\mathrm{B}_{2} \mathrm{O}_{3}(10 \% \mathrm{wt})-\mathrm{SiO}_{2}(15 \% \mathrm{wt})\right]$ with additional $2 \%$ of $\mathrm{Al}_{2} \mathrm{O}_{3}$ to avoid crystallisation. For two different $\mathrm{RuO}_{2}$ grain diameters, $40 \mathrm{~nm}$ and $400 \mathrm{~nm}$, we have realised several samples with $\mathrm{RuO}_{2}$ volume fraction $x$ ranging from about $20 \%$ down to $4 \%$. The pastes were screen printed on $96 \% \mathrm{Al}_{2} \mathrm{O}_{3}$ substrates with gold electrical contacts in order to minimise termination effects and fired for 15 minutes at various temperatures $T_{\mathrm{f}}$. The mean resistivity has been determined from $1.5 \mathrm{~mm}$ wide resistors with 8 different lengths ranging from $0.3 \mathrm{~mm}$ up to $5 \mathrm{~mm}$ and thickness of about $10 \mu \mathrm{m}$. To measure the piezoresistive response, the $\mathrm{RuO}_{2}$-glass pastes were screen printed in a Wheatstone bridge geometry on $96 \% \mathrm{Al}_{2} \mathrm{O}_{3}$ cantilever bars $51 \mathrm{~mm}$ long, $b=5 \mathrm{~mm}$ large, $h=0.63 \mathrm{~mm}$ thick, and with a reduced Young modulus of $\mathrm{E}=333$ $\mathrm{GPa}$. The cantilever was clamped at one end, whereas on the opposite end different known forces $F$ were applied at a distance $d=27.8 \mathrm{~mm}$ from the resistor. In this way, we could deduce the surface strain $\varepsilon=6 F \cdot d /\left(E \cdot b \cdot h^{2}\right)$ along the main cantilever axis, which is transferred to the TFR.

\section{RESULTS AND DISCUSSION}

In Fig. 1 we show the room temperature resistivity $\rho$ as a function of $T_{\mathrm{f}}$ for the $400 \mathrm{~nm}$ (a) and $40 \mathrm{~nm}$ (b) cases and for several values of $x$. Independently of the $\mathrm{RuO}_{2}$ grain size, $\rho$ depends only weakly on $T_{\mathrm{f}}$ for large $\mathrm{RuO}_{2}$ concentrations, while for lower values of $x$ a maximum is discernible at firing temperatures not much larger than the temperature of fusion of the glass (about $500^{\circ} \mathrm{C}$ ). In this region, the position of the maximum shifts at lower $T_{\mathrm{f}}$ values as the concentration $x$ is lowered. For even lower values of $\mathrm{x}$, the resistivity exceeds the maximum measurable value (about $10^{3} \Omega \mathrm{m}$ ) for $T_{\mathrm{f}}<600^{\circ} \mathrm{C}$. This behavior and in particular the presence of a maximum of $\rho$ has already been reported by other authors, ${ }^{3}$ and it is probably due to enhanced intergrain tunnelling distances driven by penetration of the glassy phase between the $\mathrm{RuO}_{2}$ grains.

In Fig. 2(a) we plot the resistivity as a function of $\mathrm{RuO}_{2}$ volume concentration $x$ for representative values of $T_{\mathrm{f}}$ and for both $\mathrm{RuO}_{2}$ grain diameters (see caption for more details). The blending curves display a strong enhancement of $\rho$ as $x$ is reduced and asymptotically approach infinite as $x$ becomes equal to a critical value $x_{\mathrm{c}}$. This 
behaviour is typical of a percolation transition according to which $\rho$ versus $x$ can be expressed as:

$\rho \cong \rho_{0}\left(x-x_{c}\right)^{-t}$

where $\rho_{0}$ is a prefactor and $t$ is the transport critical exponent. ${ }^{7,8}$ As shown in the $\ln -\ln$ plot in Fig. 2(b), our resistivity data follow the power law behaviour of Eq.(1) from which we can extract the fitting values of $x_{\mathrm{c}}$ and $\mathrm{t}$ reported in the figure. According to the standard percolation theory, the percolation threshold $x_{\mathrm{c}}$ is material dependent while the value of the exponent $t$ is expected to be $t=2$ for any three-dimensional system, ${ }^{9}$ regardless of the composition and the microstructure. ${ }^{7,8}$ Such universality property is due to the unimportance of microscopic details for the flow of current compared to the topological properties of the percolating backbone. In our data, only the series A1 (filled squares) fulfils the universality property while all the other samples display values of $\mathrm{t}$ much larger than $t=2$. Note that for the $400 \mathrm{~nm}$ systems, the value of $\mathrm{t}$ seems to be linked to the firing temperature. In fact, when $T_{\mathrm{f}}$ is slightly larger than the glass melting temperature the transport exponent has universal value (series A1), while for sufficiently larger $T_{\mathrm{f}}$ the transport exponent becomes nonuniversal (series A2). This correlation is less evident for the $40 \mathrm{~nm}$ series.

The lack of universality is observed also in a vast class of different materials, ${ }^{10,11,12}$ and suggests that the exponent $t$ acquires a dependence upon some microscopic properties whose nature is still unknown. ${ }^{13,14,15,16}$ To unveil the origin of nonuniversality a possible route is to apply an external perturbation with the hope of affecting the same microscopic properties governing the universality breakdown. We expect that a properly chosen applied perturbation would change the value of the critical exponent $t$.

Since in $\mathrm{RuO}_{2}$ TFRs the tunnelling between adjacent $\mathrm{RuO}_{2}$ particles plays the most important role in transport, ${ }^{3,4,5}$ then it is natural to consider the tunnelling distance a as the microscopic variable which could affect universality. An imposed mechanical strain $\varepsilon$ can modify the tunnelling distance, $a \rightarrow a(1+\varepsilon)$, and its effect on transport can be studied by measuring the piezoresistive response $\Gamma$ defined as:

$$
\Gamma=\frac{\mathrm{d} \ln (\rho)}{\mathrm{d} \varepsilon} \cong \frac{\Delta \rho}{\varepsilon \rho}
$$

where in the last term we have reported the practical definition of $\Gamma$ which is obtained experimentally by recording the resistivity change $\Delta \rho$ when an external strain $\varepsilon$ is applied to the system.

The measurements of $\Gamma$ were obtained by cantilever bar experiments with different applied forces (strains) as described in the previous section. In the whole range of applied strains (up to $\varepsilon \approx 4.5 \times 10^{-4}$ ) $\Delta \rho / \rho$ changed linearly with $\varepsilon$, permitting a rather accurate measure of $\Gamma$ from the slopes of the linear fits of $\Delta \rho / \rho v s$. $\varepsilon$. The resulting $\Gamma$ values are plotted in Fig. 3(a) for the same series of Fig. 1 as a function of the $\mathrm{RuO}_{2}$ volume fraction $x$. Apart form the "universal" A1 series, whose piezoresistive 
response remains almost constant, all the other series display a strong dependence of $\Gamma$ upon $x$, and tend to diverge as $x$ approaches to the same critical concentrations $x_{\mathrm{c}}$ at which $\rho$ goes to infinity. This behaviour can be discussed in terms of Eq.(1) which, after derivation with respect to $\varepsilon$ and according to the definition of Eq.(2), reduces to:

$$
\Gamma=\Gamma_{0}-\frac{d x}{d \varepsilon} \frac{t}{x-x_{c}}-\frac{d t}{d \varepsilon} \ln \left(x-x_{c}\right)
$$

where $\Gamma_{0}=\mathrm{d} \ln \left(\rho_{0}\right) / \mathrm{d} \varepsilon$. The second term of Eq.(3) derives from assuming that the $\mathrm{RuO}_{2}$ concentration $\mathrm{x}$ can be altered by the applied strain. However, in percolation theory, $x$ is actually a measure of the concentration $p$ of tunnelling intergrain junctions with finite resistances present in the sample. Current can flow from one end to another of the composite as long as a macroscopic cluster of junctions spans the entire sample. A small strain $\varepsilon$ can then change the values of the tunnelling resistances but cannot modify the concentration $p$ of junctions. ${ }^{17}$ According to this reasoning, the second term of Eq.(3) is then zero because $\mathrm{d} x / \mathrm{d} \varepsilon=0$. Therefore the only term which carries the $x$-dependence is the last one of Eq.(3), provided that $\mathrm{d} t / \mathrm{d} \varepsilon \neq 0$, which leads to:

$$
\Gamma=\Gamma_{0}-B \ln \left(x-x_{c}\right)
$$

where we have defined $B=\mathrm{d} x / \mathrm{d} \varepsilon$. In Fig.3(b) we report the same data of Fig. 3(a) in a semi-logarithmic plot together with fits to Eq.(4) reported as straight lines. For the A2, $\mathrm{B} 1$ and $\mathrm{B} 2$ series the data display a clear logarithmic divergence at $x_{\mathrm{c}}$ as predicted by Eq.(4) . On the contrary, the series A1 has vanishing value of $B$, in perfect agreement therefore with the universal value of its exponent $t(t \approx 2.15)$. Similar conclusions can be drawn when we analyse previously published data ${ }^{18}$ by using Eq.(4). ${ }^{17} \mathrm{We}$ can conclude therefore that when the transport exponents are non-universal, they can be modified by an applied strain.

Our findings support a tunnelling-percolation theory of universality breakdown proposed some time ago by Balberg. ${ }^{15}$ According to this model, when the distribution function of the tunnelling distance between two neighbouring grains decay much slower than the tunnelling decay, then the transport exponent reduces to:

$$
t=\left\{\begin{array}{cll}
2 & \text { if } & v+2 a / \xi \leq 2 \\
v+2 a / \xi & \text { if } & v+2 a / \xi>2
\end{array}\right.
$$

Where $a$ is the mean tunnelling distance, $\xi$ is the tunnelling decay length, and $v \approx 0.88$ is the correlation length exponent (see also Refs. 13, 19, 20). In view of Eq.(5) and Eq.(4), when $v+2 a / \xi \leq 2$ transport is universal $(t=2)$ and $B=0$, while when the mean tunnelling distance is such that $v+2 a / \xi>2$, then the critical exponent $t$ is larger than 2 and $B \equiv \mathrm{d} t / \mathrm{d} \varepsilon=2 a / \xi>0$. Both these two features are realised in our TFRs series for 
which $B=0$ when $t \approx 2$ and $B>0$ when $t>2$. Note that the sign of $\Gamma_{0}$, Eq.(3), changes from positive to negative when the critical exponent becomes non-universal (see Fig. $3 b)$. This behaviour can be explained as follows. In general, a tensile strain $(\varepsilon>0)$ enhances the inter-grain tunnelling resistances leading to an overall enhancement of the sample resistivity, so that $\Gamma=\mathrm{d} \ln (\rho) / \mathrm{d} \varepsilon$ must be positive. Hence when $B=0$, from Eq.(4) we obtain $\Gamma_{0}>0$ while when $\mathrm{B}>0, \Gamma_{0}$ does not need to be positive to ensure $\Gamma>0$. Calculations of the piezoresistive response based on the Balberg model confirm this feature. ${ }^{17}$

It is worth to point out that in addition to the importance of our results concerning the origin of transport non-universality in tunnelling-percolation materials, the reported logarithmic divergence of $\Gamma$ has also consequences for applications of Ru-based TFRs in force and pressure sensors. In fact, the efficiency of piezoresistive sensors is measured by the magnitude of $\Gamma$. However the logarithmic increase of $\Gamma$ as $x \rightarrow x_{\mathrm{c}}$ is not sufficient to overcome the power-law divergence of the resistance fluctuations. ${ }^{9}$ This poses serious limitations regarding the precision of pressure/force detection. An alternative route would be to make use of the amplification effects induced by the local elastic heterogeneities by fabricating $\mathrm{RuO}_{2}$-based TFRs with softer insulating phases like low melting temperature glasses or even polymeric hosts. Research along this line is currently undertaken.

\section{CONCLUSIONS}

We highlight the electrical transport law between nano-meter metallic particles in piezoresistive pastes by modifying their concentrations and grain sizes. We observe that for the most cases the behaviour is non-universal with transport critical exponents $t$ larger than $t=2$, and we demonstrate that the electrical response can be changed by an external applied strain. The most striking feature that we observed is the straininduced modification of the exponent $t$, which we interpret as being due to a tunnelling-distance dependence of criticality as proposed a few years ago in a tunnelling-percolation theory of non-universality. Such mechanism could apply also to other random composite materials for which transport is governed by tunnelling such as carbon-black-polymer composites. Experiments on their piezoresistive response could confirm such conjecture.

\section{ACKNOWLEDGMENTS}

This work was partially supported by TOPNANO 21 (project NAMESA, No. 5557.2). 


\section{REFERENCES}

1. Morten, B. \& Prudenziati, M., Piezoresistive thick-film sensors. in Thick Film Sensors, ed. M. Prudenziati. Elsevier, Amsterdam, 1994, pp.189-208.

2. White, N. M. \& Turner, J. D., Thick-film sensors: past, present and future. Meas. Sci. Technol., 1997, 8, 1-20.

3. Chiang, Y.-M., Silverman, L. A., French, R. H. \& Cannon, R. M. Thin glass film between ultrafine conductor particles in thick-film resistor. J. Am. Ceram. Soc., 1994, 77, 1143-1152.

4. Pike, G. E. \& Seager, C. H. Electrical properties and conduction mechanism of Ru-based thick-film (cermet) resistors. J. Appl. Phys., 1977, 48, 5152-5169.

5. Canali, C., Malavasi, D., Morten, B. Prudenziati, M. \& Taroni, A. Piezoresistive effect in thickfilm resistors. J. Appl. Phys., 1980, 51, 3282-3288.

6. Kusy, A., Classical percolation threshold and resistance versus temperature behaviour of $\mathrm{RuO}_{2-}$ glass films, Physica B, 1997, 240, 226-241.

7. Stauffer, D. \& Aharony, A., Introduction to Percolation Theory (Taylor \& Francis, London, 1994).

8. Sahimi, M., Heterogeneous Materials I: Linear Transport and Optical Properties (Springer, New York, 2003).

9. Batrouni, G. G., Hansen, A. \& Larson, B., Current distribution in the three-dimensional random resistor network at the percolation threshold. Phys. Rev. E, 1996, 53, 2292-2297.

10. Nan, C.-W., Physics of inhomogeneous inorganic materials, Prog. Mater. Sci., 1993, 37, 1-116.

11. Dziedzic, A., Percolation theory and its application in materials science and microelectronics (Part II-Experiments and numerical simulations). Inform. MIDEM, 2001, 31, 141-152.

12. Wu, J. \& McLachlan, D. S., Percolation exponents and thresholds obtained from the nearly ideal continuum percolation system graphite-boron nitride. Phy. Rev. B, 1997, 56, 1236-1248.

13. Kogut, P. M. \& Straley, J. P., Distribution-induced non-universality of the percolation conductivity exponent. J. Phys. C: Solid State Phys., 1979, 12, 2151-2159.

14. Halperin, B. I., Feng, S. \& Sen, P. N., Differences between lattice and continuum percolation transport exponents. Phys. Rev. Lett., 1985, 54, 2391-2394.

15. Balberg, I., Tunneling and nonuniversal conductivity in composite materials. Phys. Rev. Lett., 1987, 59, 1305-1308.

16. Heaney, M. B., Measurement and interpretation of nonuniversal critical exponents in disordered conductor-insulator composites. Phys. Rev. B, 1995, 52, 12477-12480.

17. Grimaldi, C., Maeder, T., Ryser, P. \& Strässler, S. Critical behavior of the piezoresistive response in $\mathrm{RuO}_{2}$-glass composites. J. Phys. D: Appl. Phys., 2003, 36, 1341-1348.

18. Carcia, P. F., Suna, A. \& Childers, W. D., Electrical conduction and strain sensitivity in RuO\$_ $\$$ thick-film resistors. J. Appl. Phys., 1983, 54, 6002-6008.

19. Stenull, O. \& Janssen, H.-K., Conductivity of continuum percolating systems. Phys. Rev. E, 2001, 64, 056105.

20. Alava, M. \& Moukarzel, C. F., Transport on percolation clusters with power-law distributed bond strength. Phys. Rev. E, 2003, 67, 056106. 


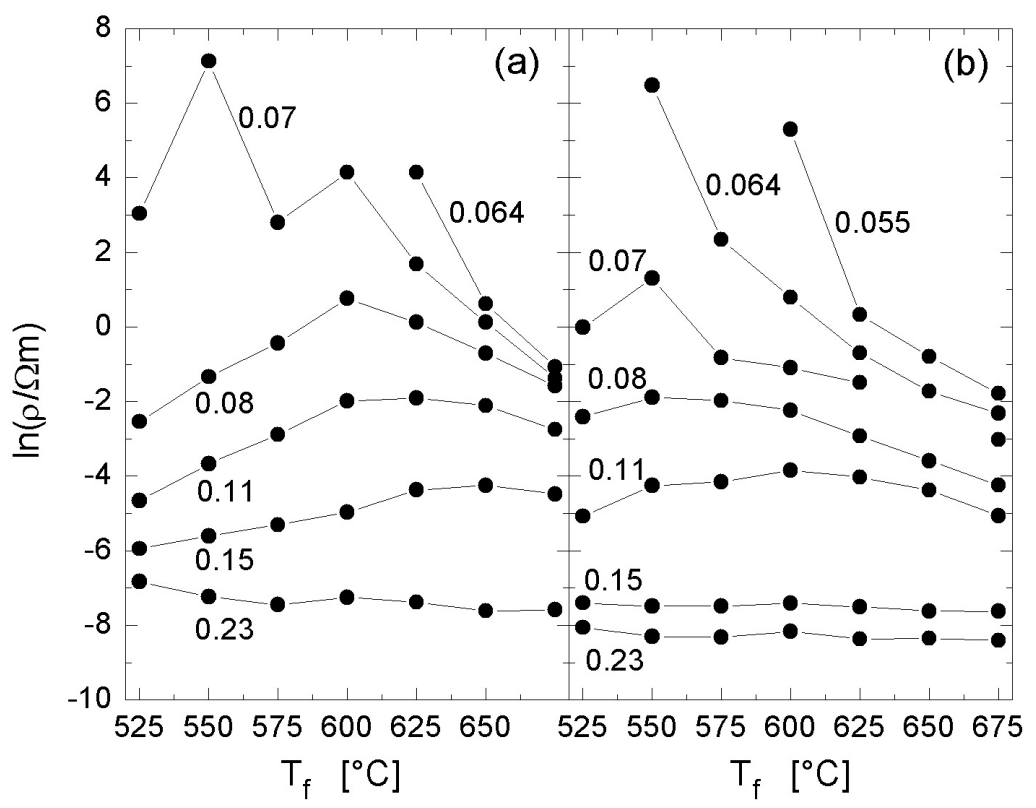

FIGURE 1. Resistivity values as a function of the firing temperature $T_{\mathrm{f}}$ for $400 \mathrm{~nm}$ (a) and $40 \mathrm{~nm}$ (b) $\mathrm{RuO}_{2}$ grain size and for different values of the $\mathrm{RuO}_{2}$ volume concentration $x$.
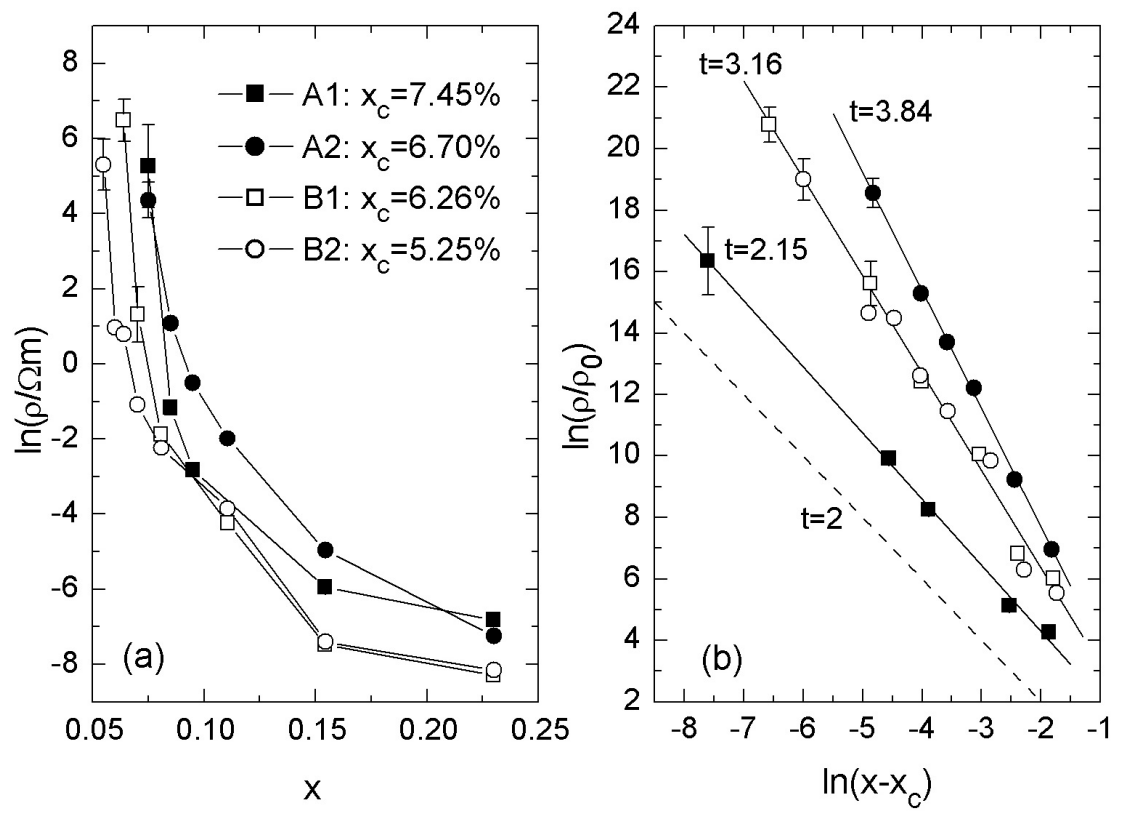

FIGURE 2. (a): resistivity $\rho$ as a function of $\mathrm{RuO}_{2}$ volume concentration $x$ for various thick-films. Labels A1 and A2 refer to samples with $400 \mathrm{~nm}$ grain size and firing temperature $T_{\mathrm{f}}=525^{\circ} \mathrm{C}$ and $T_{\mathrm{f}}=600^{\circ} \mathrm{C}$, respectively. Labels $\mathrm{B} 1$ and $\mathrm{B} 2$ refer to samples with $40 \mathrm{~nm}$ grain size and firing temperature $T_{\mathrm{f}}=550^{\circ} \mathrm{C}$ and $T_{\mathrm{f}}=600^{\circ} \mathrm{C}$, respectively. (b) ln-ln plot of the same data of (a) pointing out the power law behaviour predicted by Eq.(1) and reported by straight lines. The fitted values of the critical exponent are reported in the figure together with the expected universal value $t=2$ (dashed line). 

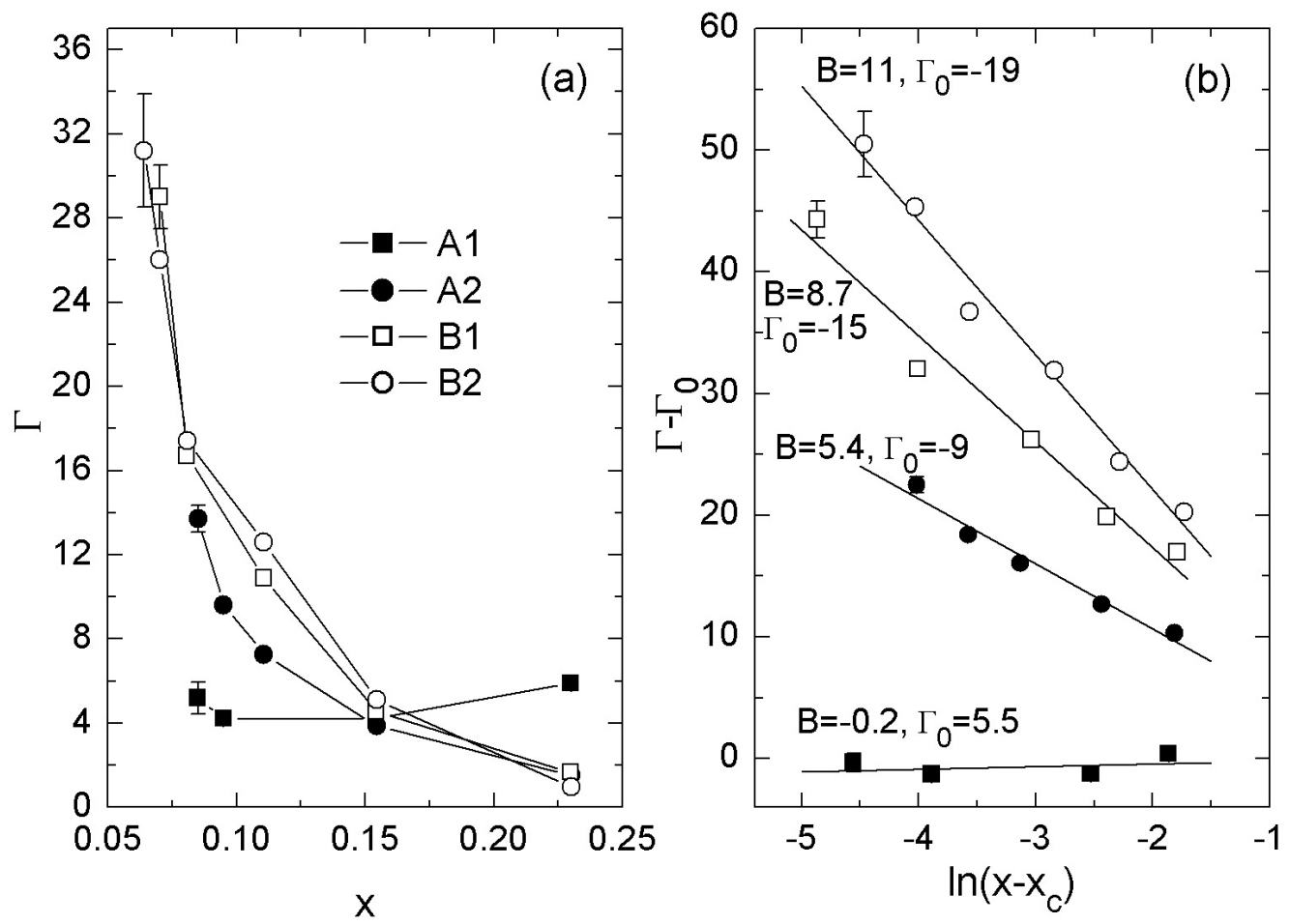

FIGURE 3. (a) piezoresistive factor $\Gamma$ as a function of $\mathrm{RuO}_{2}$ volume concentration $x$ for the same TFR series of Fig. (2). (b) semi-logarithmic plot of the same data of (a). Solid lines are fits to Eq.(4) with fitting parameters reported in the figure. 